\title{
Antropometria e aptidão física: comparação entre praticantes e não praticantes de escolinhas esportivas
}

\author{
Anthropometric and physical fitness: comparison between practitioners and non- \\ practitioners of sports schools
}

\section{Antropometría y la condición física: la comparación entre los profesionales y no profesionales de escuelas deportivas}

\author{
Anderson MIODUTZKI ${ }^{1}$ \\ William Cordeiro de SOUZA ${ }^{2}$ \\ Marcos Tadeu GRZELCZAK ${ }^{3}$ \\ Luis Paulo Gomes MASCARENHAS \\ ${ }^{1}$ Graduado em Educação pela Universidade do Contestado - UnC, Mafra - SC, Brasil \\ ${ }^{2}$ Especialista em Fisiologia do Exercício com Ênfase em Treinamento Esportivo \\ ${ }^{3}$ Mestre em Desenvolvimento Regional. Professor da Universidade do Contestado - UnC, Porto União - SC, Brasil \\ ${ }^{4}$ Doutor em Saúde da Criança e do Adolescente. Professor do Programa de Mestrado em Desenvolvimento Comunitário, \\ Unicentro - Irati - PR, Brasil
}

\begin{abstract}
Resumo
A prática regular da atividade física contribui para o desenvolvimento da aptidão física e previne a obesidade. Sendo assim, no presente estudo objetivou-se comparar as características antropométricas e de aptidão física relacionada à saúde entre crianças praticantes e não praticantes de escolinhas esportivas. A amostra foi constituída por 60 alunos de ambos os gêneros, com idade entre 7 e 12 anos da cidade de São Bento do Sul - SC, os quais foram divididos em dois grupos: 30 praticantes e 30 não praticantes regulares de exercícios físicos. Na avaliação antropométrica coletou-se a massa corporal e estatura para calculo do índice de massa corporal (IMC). Para obtenção das aptidões físicas foi avaliada a flexibilidade, o testes de força (resistência abdominal) e por fim, o teste de resistência cardiorrespiratória (6 minutos). Os testes foram realizados atendendo as recomendações sugeridas pelo PROESP-BR. Os dados foram analisados através de estatística descritiva de média e desvio padrão. Foi aplicado o Teste $t$ de Student no comparativo entre praticantes e não praticantes e o teste de qui-quadrado para determinação de diferenciação no percentual de classificação, com nível de significância estabelecido em ( $p<0,05)$. Os resultados apresentados demonstram que o grupo praticante apresentou diferença significativa apenas na variável antropométrica de estatura e na aptidão física de força (resistência abdominal) quando comparado ao grupo não praticante. Desta forma, pode-se concluir que apenas a estatura e a força (resistência abdominal) dos praticantes diferem estatisticamente do grupo não praticante. As demais variáveis apresentaram valores estatisticamente semelhantes.
\end{abstract}

Descritores: Antropometria; Aptidão Física; Exercício.

\begin{abstract}
Regular physical activity contributes to the development of physical fitness and prevents obesity. Therefore this study aimed to compare in the anthropometric characteristics and physical fitness and health among children practicing and non-practicing of sports schools. The sample consisted of 60 students of both sexes; aged 7 and 12 years of São Bento do Sul - SC, which were divided into two groups: 30 and 30 not practicing regular physical exercise practitioners. In anthropometric measurements collected to body weight and height to calculate body mass index (BMI). To obtain the physical ability was evaluated flexibility, strength tests (resistance abdominal) and, finally, the cardiorespiratory endurance testing (6 minutes). The tests were performed considering the recommendations suggested by PROESP-BR. Data were analyzed using descriptive statistics of mean and standard deviation. We applied the Student $\mathrm{t}$ test, comparing practitioners and non-practitioners and the chi-square test for determination of differentiation in the rating percentage, with a significance level in established ( $p<0.05$ ). The results show that the practitioner group showed a significant difference only in the anthropometric variable height and physical fitness strength (abdominal strength) compared to non-practicing group. Thus, it can be concluded that only the height and strength (resistance abdominal) of practitioners not differ statistically from the practitioner group, other measured variables in similar values were found.
\end{abstract}

Descriptors: Anthropometry; Physical Fitness; Exercise.

\section{Resumen}

La actividad física regular contribuye al desarrollo de la condición física y previene la obesidad. Por lo tanto, este estudio tuvo como objetivo comparar las características de aptitud antropométricas y físicas relacionadas con la salud entre los profesionales de los niños y los no practicantes de escuelas deportivas. La muestra estuvo constituida por 60 estudiantes de ambos sexos, de entre 7 a 12 años de São Bento do Sul - SC, el cual se divide en dos grupos: 30 profesionales y 30 practicantes de ejercicio físico no regulares. La evaluación antropométrica recogida con el peso corporal y la altura para calcular el índice de masa corporal (IMC). Para obtener la capacidad física se evaluó la flexibilidad, la resistencia a las pruebas de resistencia (abdominales) y, por último, la prueba de resistencia cardiorrespiratoria (6 minutos). Se llevaron a cabo los testes teniendo en cuenta las recomendaciones sugeridas por PROESP-BR. Los datos fueron analizados utilizando estadística descriptiva de la media y la desviación estándar. prueba t de Student en la comparación entre los profesionales y los no profesionales y la prueba de chi-cuadrado se utilizó para determinar la diferenciación en el porcentaje de calificación, con un nivel de significación de $(\mathrm{p}<0,05)$. Los resultados muestran que el grupo practicante mostró una diferencia significativa sólo en la altura variable antropométrica y la fuerza de aptitud física (fuerza abdominal) en comparación con el grupo no practicantes. Por lo tanto, podemos concluir que sólo se encontraron la estatura y la fuerza (fuerza abdominal) practicantes difieren estadísticamente de grupo no practicantes, en otras variables medidas valores similares.

Descriptores: Antropometría; La Aptitud Física; Ejercicio. 


\section{INTRODUÇÃO}

Atualmente, com a diminuição da prática de atividade física por crianças e adolescentes, tem-se observado um aumento gradativo da prevalência de sobrepeso e obesidade $^{1,2}$, consequentemente, essa epidemia, está apresentando relações significativas com as aptidões físicas de escolares ${ }^{3-5}$.

Segundo a Sociedade Brasileira de Medicina do Esporte $^{6}$ a prática de atividade física realizada por crianças e adolescentes melhora do perfil lipídico e metabólico, pois colabora na prevenção da obesidade, e contribui na manutenção dos níveis de aptidão física. A infância é considerada como o melhor período para o aprendizado e prevenção de inúmeras doenças, tais como; as coronarianas e a obesidade ${ }^{7}$.

Sendo assim, é de suma relevância estimular a prática de atividade física nas fases da primeira e segunda infância, bem como na adolescência, além de beneficiar qualidade de vida e saúde, a mesma endossaria a prevenção do sedentarismo na fase adulta ${ }^{8}$. Cabe mencionar que atualmente, os níveis de aptidão física de crianças e adolescentes não atingem níveis satisfatórios para a manutenção da saúde e para desempenho esportivo?.

O Colégio Americano de Medicina do Esporte $(\mathrm{ACSM})^{10}$ salienta que existem duas vertentes da aptidão física: a primeira é a aptidão física relacionada à capacidade atlética, que possui como componentes o equilíbrio, o tempo de reação, a coordenação, a agilidade, a velocidade e a potência; e a segunda vertente é a aptidão física relacionada à saúde, que é composta de cinco componentes igualmente importantes. São elas: a aptidão cardiorrespiratória, a composição corporal, a flexibilidade, a força muscular e a resistência muscular.

Dessa forma, no presente estudo objetivou-se comparar as características antropométricas e de aptidão física relacionada à saúde entre crianças praticantes e não praticantes de escolinhas esportivas.

\section{MATERIAL E MÉTODO}

\section{- Amostra}

A amostra intencional foi constituída por 60 alunos de ambos os gêneros, com idade entre 7 e 12 anos da cidade de São Bento do Sul - SC (Brasil), os quais foram divididos em dois grupos: 30 praticantes de escolinhas esportivas regulares (voleibol e futebol) e o outro grupo foi composto por 30 indivíduos não praticantes regulares de exercícios físicos. Os pais foram informados sobre o propósito da investigação, bem como sobre os procedimentos a serem adotados, e, assim esclarecidos, posteriormente assinaram o Termo de Consentimento Livre Esclarecido. Este estudo foi realizado segundo a legislação em vigor cumprindo as "Diretrizes e Normas Regulamentadoras de Pesquisa Envolvendo Seres Humanos", tendo sido aprovado pelo Comitê de Ética em Pesquisa da Universidade do
Contestado, sob o parecer $\left(\mathrm{n}^{\mathrm{o}} 1.247 .763\right.$ - CAEE: 49487115.2.0000.0117).

Como critério de inclusão, o grupo praticante foi composto somente por indivíduos com um mínimo préestabelecido de exercício físico regular de duas vezes por semana, por mais de três meses. Já o grupo não praticante foi composto por escolares que não estavam vinculados a qualquer programa de exercício físico regular?

\section{- Instrumentos e Procedimentos}

A massa corporal foi avaliada utilizando uma balança digital, com precisão de $0,1 \mathrm{~kg}$. Na medida de estatura foi utilizada uma fita métrica com precisão de $0,1 \mathrm{~cm}$ fixada na parede e no auxilio da leitura utilizouse dispositivo em forma de esquadro. A avaliação foi feita com o avaliado em apneia inspiratória, seguindo como proposto pelo PROESP-BR (Projeto Esporte Brasil $)^{11}$. A partir das informações de massa corporal e estatura, foi calculado o índice de massa corporal (IMC). Os avaliados foram classificados de acordo com Conde e Monteiro ${ }^{12}$.

A realização dos testes de aptidão física seguiu a padronização de acordo com o manual de aplicação e testes do PROESP-BR ${ }^{11}$. Foi aplicada a mesma metodologia para ambos os grupos (praticantes e não praticantes), com mesmos materiais e período do dia (vespertino), para minimizar o máximo possível as variáveis que poderiam interferir na comparação dos dados. Antes da aplicação dos testes foi realizado um aquecimento, seguindo a sugestão do PROESP-BR ${ }^{5}$.

Os alunos investigados foram submetidos à bateria de testes motores obedecendo à seguinte sequência: sentar e alcançar, como indicador da flexibilidade; abdominal, como indicador de força/resistência abdominal e por fim, corrida/caminhada de 6 minutos, para verificar o nível de aptidão cardiorrespiratória.

Com o intuito de avaliar a flexibilidade, o teste de sentar e alcançar foi empregado com a utilização do Banco de Wells. No teste de resistência abdominal, o avaliado se posicionou em decúbito dorsal com os joelhos flexionados e com os braços cruzados sobre o tórax; ao sinal, o aluno iniciou os movimentos de flexão do tronco até tocar com os cotovelos nas coxas, retornando a posição inicial, considerando o maior número de repetições completas em 1 minuto. $\mathrm{Na}$ avaliação da aptidão cardiorrespiratória dos alunos, utilizou-se o teste de corrida/caminhada de 6 minutos, com um espaço retangular de $20 \times 10$ metros. Os alunos foram informados sobre a execução correta do teste ${ }^{11}$.

\section{- Análise Estatística}

Os dados foram analisados através de estatística descritiva de média e desvio padrão. Na comparação entre os grupos, foi empregado o Teste t de Student para amostras independentes e aplicado o teste de 
homogeneidade de Levene. O teste de qui-quadrado foi empregado para comparação das porcentagens, com nível de significância estipulado em 0,05. A análise estatística foi realizada no programa SPSS Statistics 18.

\section{RESULTADOS}

A Tabela 1 apresenta os valores antropométricos e de aptidão física relacionados à saúde e obtidos no presente trabalho. Por meio dos dados obtidos foi possível verificar que o grupo praticante de modalidades esportivas apresentou diferença significativa na variável de estatura e de força (resistência abdominal) quando comparado ao grupo não praticante.

Na Tabela 2, observa-se que o grupo praticante de escolinhas esportivas apresentou um melhor desempenho quando comparado ao grupo não praticante, mas essa melhor performance não foi representada em diferenças estatísticas.

\begin{tabular}{|c|c|c|c|c|}
\hline Variáveis & Praticantes & $\begin{array}{c}\text { Não } \\
\text { praticantes }\end{array}$ & $\mathbf{T}$ & $p$ \\
\hline Idade ( & & $9,3 \pm 1,1$ & $-1,220$ & 0,227 \\
\hline Massa Corporal (kg) & $37,00 \pm 12,20$ & $34,79 \pm 10,05$ & $-0,764$ & 0,223 \\
\hline Estatura $(\mathrm{m})$ & $1,42 \pm 0,12$ & $1,35 \pm 0,10$ & $-2,105$ & $0,01^{*}$ \\
\hline IMC $\left(\mathrm{kg} / \mathrm{m}^{2}\right)$ & $17,89 \pm 3,45$ & $18,30 \pm 3,60$ & 0,453 & 0,326 \\
\hline Flexibilidade $(\mathrm{cm})$ & $32,1 \pm 4,75$ & $31,3 \pm 7,23$ & $-0,506$ & 0,307 \\
\hline Resistência Abdominal (repetições) & $33,16 \pm 8,15$ & $29,56 \pm 6,37$ & $-1,853$ & $0,03^{*}$ \\
\hline Resistência Cardiorrespiratória (m) & $954,1+86,18$ & $955,1+120,34$ & 0,017 & 0,492 \\
\hline
\end{tabular}

Tabela 2. Aptidão física relacionada à saúde

\begin{tabular}{|c|c|c|}
\hline Variáveis & $\begin{array}{c}\text { Praticantes (\%) } \\
(n=30)\end{array}$ & $\begin{array}{c}\text { Não Praticantes }(\% \\
(\mathbf{n}=\mathbf{3 0})\end{array}$ \\
\hline \multicolumn{3}{|l|}{ IMC } \\
\hline Zona Saudável & $\mathrm{n}=21(70 \%)$ & $\mathrm{n}=22(73,33 \%)$ \\
\hline \multicolumn{3}{|l|}{ Flexibilidade } \\
\hline Zona Saudável & $\mathrm{n}=15(50 \%)$ & $n=13(43,33 \%)$ \\
\hline Zona de Risco à Saúde & $\mathrm{n}=15(50 \%)$ & $n=17(56,67 \%)$ \\
\hline \multicolumn{3}{|l|}{ Resistência Abdominal } \\
\hline Zona Saudável & $\mathrm{n}=24(80,00 \%)$ & $n=24(80,00 \%)$ \\
\hline Zona de Risco à Saúde & $\mathrm{n}=6(20,00 \%)$ & $\mathrm{n}=6(20,00 \%)$ \\
\hline \multicolumn{3}{|l|}{ Resistência Cardiorrespiratória } \\
\hline Zona Saudável & $\mathrm{n}=29(89,66 \%)$ & $\mathrm{n}=22(70,33 \%)$ \\
\hline Zona de Risco à Saúde & $n=1(10,34 \%)$ & $\mathrm{n}=8(22,67 \%)$ \\
\hline
\end{tabular}

\section{DISCUSSÃO}

Por meio dos resultados obtidos no presente estudo foi possível verificar diferenças significativas somente na estatura e resistência abdominal. Para as outras variáveis mensuradas não foram encontradas diferenças significativas. Vale ressaltar que quando comparadas as proporções obtidas nas classificações do IMC, flexibilidade, resistência abdominal e aptidão cardiorrespiratória também não foram observadas diferenças significativas entre os grupos avaliados, tanto na zona saudável como na zona de risco à saúde.

A composição corporal não apresentou diferenças significativas entre os grupos avaliados. Em estudo desenvolvido por Cyrino et al. ${ }^{13}$ que compararam o IMC entre jovens praticantes e não praticantes de futsal durante um período de 24 semanas de treinamento de futsal, com 3 sessões semanais de treinamento da modalidade, em dias alternados, com duas horas e meia de duração também não foi observada alteração significativa no IMC dos voluntários. Resultados semelhantes foram encontrados no presente estudo.

De acordo com o $\mathrm{ACSM}^{14}$ três sessões semanais de treinamento aeróbico moderado, ou seja, entre 50 a $80 \%$ da frequência máxima cardíaca (FCmáx), com duração aproximada de 30 minutos, pode ser suficiente para controlar o peso corporal e, por conseguinte, o IMC. Desse modo, ao analisar os resultados do presente estudo e considerar as diretrizes propostas pelo $\mathrm{ACSM}^{14}$, pode ser que o processo de treinamento ao qual o grupo de crianças que participam de escolas de esporte é submetido, não seja suficiente para interferir de maneira significativa no IMC dessas crianças.

Já a flexibilidade não apresentou diferença significativa entre os grupos para esta variável. Segundo o manual do PROESP Brasil ${ }^{11}$ os valores devem estar na faixa de 28,9 a 39,5 para a faixa entre 7 e 12 anos de ambos os sexos. Nos testes foram observados que os valores alcançados estão na faixa recomendada para a saúde. A ausência de diferença na flexibilidade observada entre os grupos pode ser explicada pela falta de especificidade dos treinamentos realizados pelas crianças que participam de escolas de esporte.

Um fator que pode ter influenciado na falta de diferença significativa na flexibilidade é a falta de precisão do teste de sentar e alcançar. Sendo que quando comparados dois testes de flexibilidade, o teste de sentar e alcançar tradicional, no qual o ponto inicial de medição é prefixado usando o Banco de Wells, e o teste de sentar e alcançar modificado, cujo ponto inicial é ajustado de acordo com o comprimento do membro superior do sujeito. A análise dos resultados mostrou que o teste de sentar e alcançar tradicional sofre influência significativa do comprimento dos membros do sujeito submetido ao teste, isso pode ser um fator determinante, já que no presente estudo a estatura entre os dois grupos apresentou diferença significativa ${ }^{15}$.

Contrapondo-se ao presente estudo Seabra, Maia e Garganta $^{16}$ analisaram o crescimento, a maturação e a aptidão física de jovens jogadores de futebol e não jogadores de futebol de Portugal e verificaram que os resultados do estudo não mostraram diferenças significativas entre o grupo de crianças que praticavam futebol e não praticavam futebol, na variável de força/resistência abdominal.

De acordo com Glaner ${ }^{17}$ a prática regular de exercício físico influencia o desenvolvimento da capacidade de força/resistência abdominal. Além disso, alguns autores sugerem que a prática regular de exercícios físicos, como, por exemplo, a participação em escolas de esporte, pode elevar a produção hormonal de testosterona $^{18-20}$.

Dessa maneira, o aumento da produção de testosterona favorece o desenvolvimento da massa muscular nas crianças que participam desse tipo de 
atividade $^{20}$. Assim o processo de treinamento contínuo que os praticantes de escolinhas esportivas executam diariamente pode ser suficiente para interferir de maneira significativa no resultado do teste de força (resistência abdominal).

Por fim, a aptidão cardiorrespiratória que não apresentou diferença significativa no teste realizado. Um fator que pode ter influenciado para que não houvesse diferença entre os grupos é o nível de atividade física habitual das crianças não participantes de escolinhas esportivas. Infelizmente, o presente estudo limitou-se em não controlar essa variável.

De acordo com McArdle et al. ${ }^{18}$, 3 a 5 sessões semanais de treinamento aeróbico, com duração entre 20 a 30 minutos e com intensidade aproximada de 40 a $85 \%$ do $\mathrm{VO}^{2}$ pico durante um período de 10 semanas, podem causar alterações significativas na capacidade aeróbica de um indivíduo. Entretanto, ainda de acordo com esse autor, deve-se considerar o estado aeróbico inicial do sujeito para que haja maior precisão na prescrição do treinamento aeróbico.

De certa forma, a intensidade, frequência e volume de exercícios físicos impostos aos praticantes de exercícios físicos não são suficientes para aumentar/contribuir na melhora da aptidão física dessas crianças.

\section{CONCLUSÃO}

Com base na metodologia empregada e nos resultados obtidos no presente trabalho é possível concluir que apenas a estatura e a força (resistência abdominal) dos praticantes diferem estatisticamente do grupo não praticante. Nas outras variáveis mensuradas foram encontrados valores estatisticamente semelhantes.

\section{REFERÊNCIAS}

1. Rinaldi AEM, Pereira AF, Macedo CS, Mota JF, Burini RC. Contribuições das práticas alimentares e inatividade física para o excesso de peso infantil. Rev Paul Pediatr. 2008; 26(3):271-7.

2. Costa MJM, Araújo MLLM, Araújo MAM, MoreiraAraújo RSR. Excesso de peso e obesidade em préescolares e a prática de atividade física. $\mathrm{R}$ bras $\mathrm{Ci}$ e Mov. 2015; 23(3):70-80.

3. Conte M, Gonçalves A, Aragon FF, Padovani CR. Influência da massa corporal sobre a aptidão física em adolescentes: estudo a partir de escolares do ensino fundamental e médio de Sorocaba/SP. Rev Bras Med Esporte. 2000; 6(2):44-9.

4. Ferreira Junior MJ, Reuter CP, Meinhardt FP, Corbellini VA, Burgos MS. Obesidade e aptidão física relacionada à saúde: um estudo com escolares de Santa Cruz do Sul - RS. Fiep Bulletin. 2012; 82(Special Edition):1-5.

5. Mascarenhas LPG, Ferreira AB, Lima VA,
Grzelczak MT. Estudo comparativo da aptidão física entre crianças de escola pública e particular: uma visão regional. Cinergis. 2013; 14(3):157-60.

6. Sociedade Brasileira de Medicina do Esporte (Posicionamento Oficial). Atividade física e saúde na infância e adolescência. Rev Bras Med Esporte. 1998; 4(4):107-9.

7. Crippa F, La Torre M. Comparação das características antropométricas e da força muscular de meninas de 9 a 11 anos praticantes e não praticantes de exercício físico regular. Cinergis. 2013; 14(1):1-6.

8. Guyton AC, Hall JE. Tratado de fisiologia médica. $11^{\mathrm{a}}$ ed. Rio de Janeiro: Elsevier; 2006.

9. Farias JP, Faria WF, Brito LS, Marroni PCT, Elias RGM. Aptidão física de adolescentes praticantes e não praticantes de handebol. Rev Digital - Buenos Aires. 2014; 19(197):1.

10. American College of Sports Medicine. Manual do ACMS para Avaliação da Aptidão Física Relacionada à Saúde. Guanabara Koogan; 2006.

11. Projeto Esporte Brasil. manual. 2012 Disponível em: <http:// www.proesp.ufrgs.br> Acesso em: 25 de setembro 2015.

12. Conde WL, Monteiro CA. Body mass index cutoff points for evaluation of nutritional status in Brazilian children and adolescents. J Pediatr. 2006; 82(4):26672.

13. Cyrino ES, Altimari LR, Okano AH, Coelho CF. Efeitos do treinamento e futsal sobre a composição corporal e o desempenho motor de jovens atletas. $\mathrm{R}$ bras de Ci e Mov. 2002; 10(1): 41-6.

14. American College of Sports Medicine. Manual do ACMS para Avaliação da Aptidão Física Relacionada à Saúde. Rio de Janeiro: Guanabara Koogan; 2000.

15. Hoeger WWK, Hopkins DR. A comparison of the sit and reach and the modified sit and reach in measurement of flexibility in women. Research Quarterly for Exercise and Sport. 1992; 63(2): 191-5.

16. Seabra A, Maia JA, Garganta R. Crescimento, maturação, aptidão física, força explosiva $\mathrm{e}$ habilidades motoras específicas. Estudo em jovens futebolistas e não futebolistas do sexo masculino dos 12 aos 16 anos. Revista Portuguesa de Ciências do Desporto. 2001; 1(2):22-35.

17. Glaner MF. Crescimento e aptidão física relacionada à saúde em adolescentes rurais e urbanos em relação a critérios de referência. Rev Bras Educ Fís Esporte. 2005; 19(1):13-24.

18. Mcardle WD, Katch FI, Katch VL. Fisiologia do exercício: energia, nutrição e desempenho humano. 5. ed. Rio de Janeiro: Guanabara Koogan; 2003.

19. Astrand PO, Rodahl K, Dahl HA, Stromme SB. Tratado de Fisiologia do trabalho: bases fisiológicas 
do exercício. $4^{\mathrm{a}}$ ed. Porto Alegre: Artmed; 2006.

20. Generosi RA, Baroni BF, Junior ECPL, Bergmann GG, Garlipp DC, Cardoso M. Aptidão física de crianças e adolescentes escolares praticantes de esportes extracurriculares. Rev Educ Fís. 2009; 144:13-22.

\section{CONFLITO DE INTERESSES}

Os autores declaram não haver conflitos de interesse.

\section{AUTOR PARA CORRESPONDÊNCIA}

Anderson Miodutzki

iti_anderson@hotmail.com

Submetido em 18/06/2016

Aceito em 27/06/2016 